\title{
Do-It-Yourself (DIY) Integration of Paper Sensor in a Smart Lid for Medication Adherence
}

\author{
Sherjeel M. Khan ${ }^{1}$, Nadeem Qaiser ${ }^{1}$, Sohail F. Shaikh ${ }^{1}$, Lijie J. Ding ${ }^{1}$ \& Muhammad M. Hussain ${ }^{1,2 *}$ \\ ${ }^{1} \mathrm{mmh}$ labs, King Abdullah University of Science and Technology, Thuwal, Saudi Arabia. \\ ${ }^{2}$ EECS, University of California, Berkeley, California, USA.
}

Muhammadmustafa.hussain@kaust.edu.sa

\begin{abstract}
Poor adherence to prescription medications causes approximately 125,000 deaths per year while an epidemic of a prescription drug overdose has been on the rise, resulting in an alarming number of deaths, 47,055 in 2014 only in the USA. Efforts from governments to address this issue are technologically inadequate. Once a patient leaves the pharmacy with a prescription drug container, no one, besides the patient, has control over the safe use of drugs. Here, we show a Do-It-Yourself (DIY) integration strategy to monitor drug intake, using a low-cost paper sensor-based flexible electronic system integrated inside the cap of a drug container. This electronic system consists of flexible modular decal sensors connected to a central flexible decal electronic interface, using a DIY compatible integration technique using an anisotropic conductive tape. We find that our DIY integration strategy gives the flexibility to develop customized versions of the system, depending on the requirements for different applications, ranging from ambient sensors to monitor the environment inside the container to security sensors that can detect any kind of mishandling with the prescription container. The add-on system is easy to install or remove, enabling an easy implementation for practical use that will ultimately mitigate drug overuse.
\end{abstract}

Keywords: Medication Adherence; Paper Sensor; Flexible Electronics; DIY Assembly; Anisotropic conductive tape

\section{Introduction}

Over the past three decades, a global epidemic of prescription drug abuse has been on the rise. Although not necessarily ignored, no tangible solution has been found to this day. The global estimate for drug-related deaths in 2014 was over 200,000 despite the fact that drug addiction usage has been stagnant for 10 years [1]. This points to a more serious problem: the fact that these deaths are actually correlated to a growing rise in prescription drug abuse, rather than other types of drug addiction. The USA is hit the hardest, experiencing an alarming increase from 6100 deaths in 1980 to 47055 in 2014, equivalent to nearly 125 people dying every day, half of those directly attributed to prescription drug overdoses [2, 3]. NonGovernment Organizations (NGOs) have become more involved in increasing awareness and supporting stringent state laws to control this problem. However, limiting access to opioid pain relievers may result in an increase in mortality rates as people may start looking for more dangerous alternative drugs to treat their pain. Taking prescription drugs without medical supervision presents even greater risks of a fatal drug overdose. When denied, patients start to 'hop' from one doctor or pharmacy to another in order to have their prescription containers filled, and a study found that these individuals were more prone to a fatal drug overdose [4].

There is plenty of (sensing) technologies available, and researchers have been developing to build devices that can counteract diseases and epidemics. However, the implementation of these tools has been slow because of the high cost of sensors and the complexity associated with their fabrication and assembly. First, the microfabrication of sensors requires expensive equipment that can then build the sensors and in order to form a complete solution, these sensors undergo complex integration processes. For instance, Printed Circuit Boards (PCB) manufacturing involves fabricating circuit boards, and soldering sensors and processors on them. These restrictions create limitations on the availability of such devices for the low-income population, which, ironically, suffers the most from these epidemics and diseases $[5,6]$. We need simpler, low-cost solutions accessible to everyone.

We introduce the anisotropic conductive tape (ACT) as a practical, low-cost and easy-to-use potential solution. An ACT consists of conducting particles embedded in an adhesive film, such that when two conductors placed on both sides of the film, an electrical connection is formed by application of pressure or heat. Similar devices have been widely used for 
flat panel display packaging and chip-on-film assembly [7-9] Here, we present an integration strategy using an ACT to assemble low-cost paper-based, stretchable, temperature and humidity sensors, in such a way that anyone can make and assemble these sensors into a functional device without the need of any technical expertise. We further study the contact mechanics and electrical behavior of the connection between paper sensors and ACT. Using a Do-It-Yourself (DIY) approach, we assemble and further develop an affordable selfcontained pill monitoring smart lid that counts and reports pill usage, using a cell phone, and alerts in case someone forcibly tampers the container for the purpose of drug overuse. First, we convert the paper-based sensors into modular form so that these sensors can be easily installed and removed from a functional system. We then fabricate an underlying electrical circuit using conductive copper tapes and the sensors are then assembled onto the custom designed circuit to be further integrated with the central electronic interface, using ACT. Since the functionality is kept modular, paper-based temperature and humidity sensors can be attached, as needed, to monitor the ambient environment inside the prescription container, or they can be replaced by a conductive rubberbased strain sensor and proximity sensor to monitor tampering attempts on the container itself. The sensors are designed so that none of them requires an active source of power, unlike traditional commercial integrated circuit sensors. They are powered instantaneously, and only when data need to be logged. This reduces the system's power consumption to a bare minimum. Additionally, by adding a smart module, the container then has the ability to provide the location of the patient and inform authorities or caretakers via SMS/Call whenever the patient is tampering with the container or abusing the recommended dosage. This makes it possible to respond immediately and to potentially save lives.

\section{Design and Materials}

\subsection{ACT Enabled Integration}

To make it compatible with a DIY assembly, we use an ACT-based sensor assembly approach, to integrate the sensor with electronic interfaces. For the same reason, we opt for a 3M ${ }^{\mathrm{TM}}$ Electrically Conductive Adhesive Transfer Tape 9703 consisting of a $50 \mu \mathrm{m}$ acrylic adhesive with silver particles spread across, at a pitch of $400 \mu \mathrm{m}$. When placed between two conductors and with the application of finger pressure, it forms an electrical contact between the top and bottom electrodes. As shown in Figure 1a, when ACT is merely placed inbetween two conductors, these conductors do not form an electrical contact as they are not in contact with the embedded silver particles. On application of finger pressure, the two conductors are pressed towards each other, inside the adhesive, and at some point, form an electrical contact after touching the silver particles from both sides. The acrylic adhesive binds the two conductors in the same place and this allows a current to flow anisotropically from one conductor to

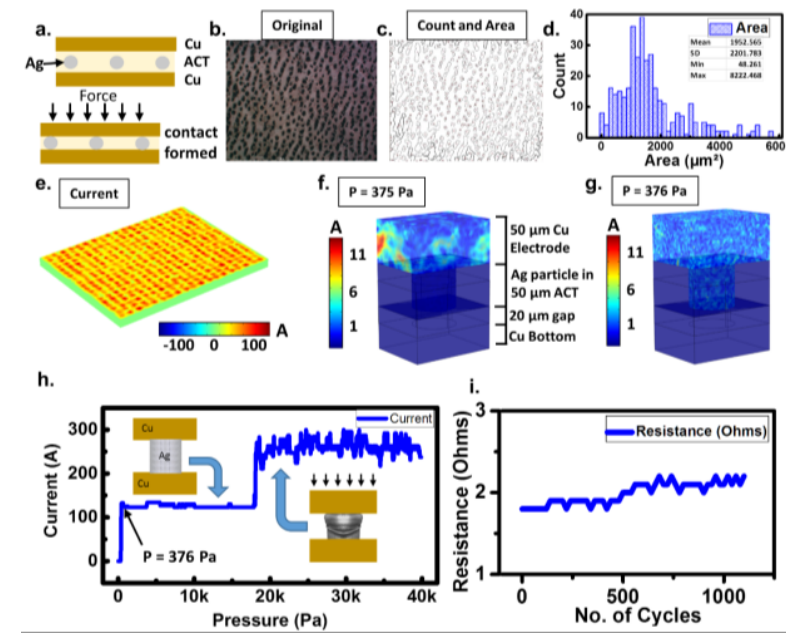

Figure 1: (a) Illustration of how the anisotropic conductive tape (ACT) conducts current between two conductors, through embedded silver particles, after application of pressure. (b) Microscopic image of $7 \mathrm{~mm}^{2}$ area of the ACT with silver particles shown in dark color; transparent adhesive is shown in light color. (c) Processed image, using ImageJ software, to count the number of particles in the sample and their surface areas. (d) Histogram of distribution of silver particles according to their surface area. (e) FEM simulation of the replica model of $7 \mathrm{~mm}^{2}$ sample showing the electric current passing through the areas where silver particles are modeled. (f) FEM simulation of contact mechanics showing that pressures below $375 \mathrm{~Pa}$ do not form electrical contact through the prescribed $20 \mu \mathrm{m}$ gap between the ACT and the copper electrode. (g) FEM simulation showing contact is formed at $376 \mathrm{~Pa}$ pressure to overcome the $20 \mu \mathrm{m}$ gap between the ACT and copper. (h) Current passing through the modeled contact for pressures between $0-40 \mathrm{kPa}$. (i) Resistance change in the contact formed between the ACT and the copper tape, using finger pressure, for $11001 \mathrm{~cm}$ bending radius cycles.

another. In order to study the electrical behavior and performance of the tape, we take a microscopic $\left(7 \mathrm{~mm}^{2}\right.$ area $)$ image of the ACT (Figure 1b). In order to count the number of particles in a given area and the average size of each particle, we process a sample of the ACT image, using Javabased "ImageJ" software. First, the image must be converted to a binary image in order to be processed and for the software to perform the required calculations. After conversion and analysis by the image processing software, we can establish the number of particles in the sample and the particle's average surface area, as shown in Figure 1c. From the data obtained, we plot the histogram of particle sizes spread across the sample (Figure 1d). We obtain a value of $2000 \mu \mathrm{m}^{2}$ for the mean surface area of particles. We further took similar measurements from 9 other samples from different areas of ACT and found out that the number of particles varied between 300-400 with an average of 360, while the area was found to be $2000 \pm 50 \mu \mathrm{m}^{2}$. The microscope used could only produce images of $7 \mathrm{~mm}^{2}$ area. Based on this information, we recreate a $7 \mathrm{~mm}^{2}$ model of the acrylic tape, with 360 silver cylindrical particles of $2000 \mu \mathrm{m}^{2}$ surface area and $50 \mu \mathrm{m}$ height, sandwiched between two copper electrodes. We then perform FEM electrical simulations with a potential of $5 \mathrm{~V}$ applied across the two copper conductors. The resulting current flow is presented in Figure 1e. In this figure, we see that the areas containing silver particles conduct current. In an 
ideal case, the electrical contact becomes ohmic, with almost zero contact resistance. This happens when the two metals are in complete contact, due to the fact that the cylindrical silver (of $50 \mu \mathrm{m}$ height and $2000 \mu \mathrm{m}^{2}$ surface area) has a negligible resistance $(0.0403 \Omega)$ of its own. The value of current obtained from simulation results $(118 \mathrm{~A})$ corroborate the mathematical value of current $(124 \mathrm{~A})$ that flows when a $5 \mathrm{~V}$ potential is applied across a silver particle of $0.0403 \Omega$.

In a real-life non-ideal scenario, when two conductors are placed around the ACT, no electrical contact can be established since the pressure is required for the silver particles to form a contact between the two conductors. To establish that pressure, we create a model of one silver particle (of $50 \mu \mathrm{m}$ height and $2000 \mu \mathrm{m}^{2}$ surface area) in between two conductors, with two conductors of $50 \times 100 \mu \mathrm{m}^{2}$ surface area (each) and a $50 \mu \mathrm{m}$ height. An SEM image of the ACT when merely placed on the copper tape showed that an approximate gap of $20 \mu \mathrm{m}$ is present when no force is exerted by a finger to secure the connection (Supplementary Figure S1a). The size of silver particles is kept identical to that used in previous simulation studies, however, a $20 \mu \mathrm{m}$ gap is now created between the top conductor and the silver particle. After performing FEM contact simulations in COMSOL, using this model, and by varying pressure and observing current conductivity, we find that electrical contact is established when a pressure of $376 \mathrm{~Pa}$ is applied. As shown in Figure 1f, for a pressure of $375 \mathrm{~Pa}$ and below, no current flows through the silver particle and remains confined in the top electrode. At $376 \mathrm{~Pa}$, a current starts to flow between the two conductors through the silver particles in the ACT (Figure 1g). From these experiments, we can conclude that a pressure of $376 \mathrm{~Pa}$ per silver particle (or per $5000 \mu \mathrm{m}^{2}$ area) is required to establish an electrical current. This corresponds to a pressure of 1.5 $\mathrm{kPa} / \mathrm{cm}^{2}$ which can be easily applied by human finger [10]. Supplementary Figure S1b shows the normal force exerted by a finger and a thumb, calculated using a commercial force sensor FSG015WNP for a fingertip area of $2 \mathrm{~cm}^{2}$. It is evident that a finger press can provide sufficient pressure to form an electrical contact using ACT. The Supplementary Video S1 shows how the electrical current starts at $376 \mathrm{~Pa}$ and increases up to $377 \mathrm{~Pa}$ after which it remains constant since electrical contact is formed at $376 \mathrm{~Pa}$, and further application of pressure will not change the current conductivity significantly. However, at higher pressures, the shape of the particles will change (deform) and surface area between the particles will increase. This occurs because the silver particle, at the top of the cylinder, becomes compressed and forms an irregular shape, as shown in Supplementary Figure S1d. At this point, the current conducting through the silver particle increases significantly. To study this behavior, we run a pressure sweep from 0 to $40 \mathrm{kPa}$, using the same FEM model as we did for finding out the pressure needed to make a contact with a 20 $\mu \mathrm{m}$ gap. We then observe the flow of the current at a fixed point on the silver particle, for different values of applied pressure (Figure 1h). The plot displayed in Figure 1h confirms the hypothesis that, after the first initial contact at $376 \mathrm{~Pa}$, the current remains almost constant until $20 \mathrm{kPa}$. However, for pressures above $20 \mathrm{kPa}$, the current suddenly increases suddenly and then oscillates. This behavior can be attributed to the deformation of the silver particle, which increases the contact area between the two electrodes (Supplementary Figure S1d). Since the material is under stress and the contact is not ideal, the current behavior is similar to a loose contact showing a current sparking phenomenon [11]. The resistance (R) of the silver particle can be expressed as:

$$
R=\frac{\rho L}{A}
$$

The increase in current is caused by the decrease in resistance $(\mathrm{R})$ of the silver particle, due to a reduction in length (L) and increase in surface area (A). Since we are using inherently flexible materials to make our sensors, electrical connections, and the substrate, we further examine the performance of the electrical contact, using ACT under bending. In order to test the performance of an electrical connection formed using the ACT, we prepared a sample to undergo cycling bending testing. The sample preparation is detailed in Supplementary Section 1. We run the sample through 1100 cycles of $1 \mathrm{~cm}$ bending radius (this value represents the median bending radius of daily events that these systems can experience), using a custom-built bending machine; we then monitor the change in resistance over time. Figure 1i shows the bending tests results from over 1100 cycles for which each resistance measurement is taken after every 20 cycles. The graph displayed in Figure 1i shows the resistance to be $2.1+0.1 \Omega$ at the end of 1100 bending cycles. Such minute changes in resistance show that, with our ACT based paper sensor integration strategy, the performance of the sensors is not affected, even after undergoing vigorous handling and bending over time.

\subsection{Smart Lid Components}

To overcome the fact that patients may forget to take a pill or may overdose, intentionally or non-intentionally, we incorporate an add-on system in the standard drug container to count and record pill usage and sync the data through patients' cell phones, allowing them to properly follow the recommended dosage. Besides being used for things, the addon approach has shown its advantages in plants and marine species monitoring. Add-on approach provides the ability to add functionality to existing beings non-intrusively [12-14]. Further benefits of our smart lid over currently available solutions are in Supplementary Section 2.

\subsubsection{Modular Paper-Based Pill Health Monitoring}

Sensor. Storage conditions are vital for the safety, integrity, and effectiveness of most medicines. Temperature and humidity are known to influence the integrity and potency of medicinal pills. Elevated temperatures and humidity fasten the degradation process of tablets $[15,16]$, and doctors recommend storage at room temperature for most medicines. 
We make use of our previous work on low-cost flexible paper sensors to monitor the ambient condition inside the bottle for pill health monitoring. As an example, any tablet containing chitosan has to be stored under $25{ }^{\circ} \mathrm{C}$ and $60 \%$ relative humidity $(\mathrm{RH})$ to avoid the degradation of its physical properties [17]. Similarly, it was shown that increased temperature and humidity severely affect the stability of ascorbic acid-based tablets, the most widely used medicine [15]. Additionally, several studies showed a very significant reduction in dissolution time for tablets under exacerbated humid conditions that can also change the appearance, hardness, mass and crystallinity of the medicine due to moisture absorption [18-20]. It is very important to monitor storage conditions inside the prescription container as they are more prone to deterioration, once removed from the foil [21]. Nassar et al. previously investigated the performance characteristics of paper-based humidity and temperature sensors for wearable applications and showed that these sensors performed consistently under various bending conditions [22]. The paper-based temperature and humidity sensors are drawn on a $3 \mathrm{M}$ post-it note using a conductive silver ink pen. Silver pen on papers showed a resistivity of $\sim 0.2 \Omega /$ sq. A serpentine pattern is drawn on the paper to act as a resistive temperature sensor. The resistance of silver ink changes in response to changes in temperature. A combed shaped capacitive structure is drawn to make the humidity sensor. The paper acts as the dielectric material for the capacitive structure. As the humidity rises and moisture vapors adsorb on the paper surface in-between the electrodes, the capacitance changes due to the change in effective dielectric constant. We, therefore, used similar paper-based sensors to monitor the ambient environment inside the container. We find that our (paper-based) temperature sensors deliver linear results from $20-100{ }^{\circ} \mathrm{C}$, which is a temperature range suitable for our study. Generally, to integrate paperbased sensors on a flexible platform, interconnected patterns of gold or copper are deposited on a flexible polyimide substrate. These sensors are connected to the electronic circuitry using epoxy or other conductive adhesives. This increases the cost and complexity of the procedure, due to the use of sophisticated equipment in cleanroom facilities and bonding methods (using epoxy) which are incompatible with large scale manufacturing. However, our DIY method avoids the use of such expensive equipment or complex procedures.

This DIY method first converts paper sensors into modular decal sensors that can be attached to our electronic interface, in a customized fashion. In order to give paper sensors their modular form, we take a paper temperature sensor and we separately cut thin long strips of conductive copper foil. These strips were then connected to electrodes on the paper sensors from one end and then folded towards the backside of the sensor, enabling the modular attachment of the sensor to the base decal. Figure 2a shows how the copper foil is in contact with the silver ink, on paper, and then wrapped around the paper to fold towards the backside of the paper. A small piece

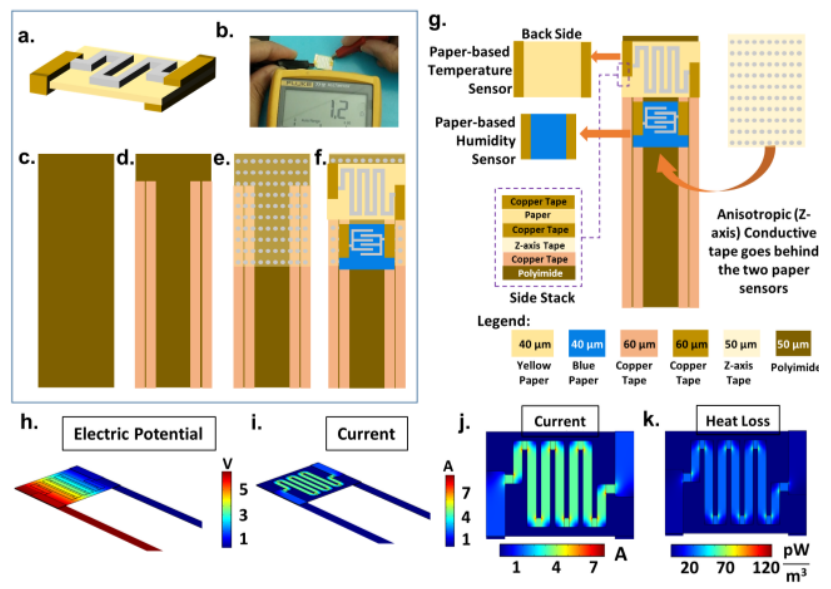

Figure 2: (a) Side view of the modular paper sensor with copper foil folding towards the back side so that the sensor can be placed on top of copper to form the electrical contact through the ACT. (b) Electrical resistance of the temperature sensor after contact is made. (c) A PI sheet taken as a substrate. (d) Copper tape interconnects formed on the PI sheet to make a "decal". (e) The ACT placed over the entire decal area where sensors are to be placed. (f) Paper-based sensors attached directly over the copper tape on PI, through the ACT. (g) Illustration of the complete decal assembly with the side stack showing the placement of each layer on top of each other. (h) Electric potential distribution over the decal with a paper temperature sensor. (i) Current flow through the temperature sensor. (j) Current flow through the temperature sensor showing high current density area around the 90-degree bends. (k) Heat loss through the temperature sensor where small pockets of heat visible at $90^{\circ}$ bends.

of Kapton Tape is used to secure the copper foil strip at the contact point, with silver, to avoid any contact resistance. The resistance of the sensor, before contact is established, is $1.2 \Omega$, and remains the same after making contact with the copper tape with Kapton tape securing the bond (Figure 2b). This shows that a zero-contact resistance can be achieved by a simple application of a Kapton tape on the copper foil at the contact point, eliminating the further need for soldering and epoxy. Similarly, we convert the paper humidity sensor into a modular form. Then, we prepare the decal, which serves both as a carrier platform to host the modular sensors and as the base substrate onto which interconnects are formed in order to enable conformal connections between the modular paper sensors and the central electronic interface. To prepare the base structure ('decal') onto which sensors will be attached, we take a Polyimide sheet (DuPont ${ }^{\mathrm{TM}}$ Kapton ${ }^{\circledR}$ 200HN Polyimide Film, 50 Micron Thickness) (Figure 2c) and prepare a pattern of interconnects, using conductive copper tape of similar dimensions as those of modular paper sensors previously made (Figure 2d). Any substrate (even a piece of paper) can be chosen as it only serves as an underlying carrier substrate. The ACT is placed in the area where the paper sensors need to be attached (Figure 2e). The function of the $\mathrm{ACT}$ is essentia 1 in order to achieve the desirable zero Ohm contact resistance between the copper tape of the paper sensors and the copper tape on the polyimide decal. After assembly of the sensors onto the substrate in a completely modular fashion, customized and flexible decal sensors to monitor humidity and temperature (Figure 2f) are created and the decal can be attached, later on, to the central electronic 
interface. Figure $2 \mathrm{~g}$ shows an illustration of the decal and a stack of modular paper sensors attached to the decal through ACT. In order to test the performance of the modular sensor assembly using ACT, we prepare a similar model of the decal with a temperature sensor in COMSOL. Figure $2 \mathrm{~h}$ shows how the potential of $5 \mathrm{~V}$ is applied to one side and the ground on the other side. The two long electrodes are the copper conductive tape on the Kapton Sheet and the paper sensor is placed on them through the ACT. For the contact modeling between ACT and copper electrodes, we used the same model discussed in Section 2. After performing FEM simulations and observing the current flow through the whole system (Figure $2 \mathrm{i}$ ), we see that a current of $4.2 \mathrm{~A}$ passes through the sensor, which corroborates with the mathematical value of current (4.16 A) passing through the $1.2 \Omega$ sensor for a $5 \mathrm{~V}$ potential. In Figure 2j, we can see the current flow closely. The current flows uniformly, albeit with a higher current density illustrated by red-yellow lines along the sharp 90 degree turns in the sensor. These losses are confined in a small area and create minute heating points that do not heat up the sensor (Figure 2k). Our DIY process of creating modular paper sensors requires minimal technical expertise, as it circumvents conventional techniques of soldering and etching used in PCB, or the microfabrication processes of metal deposition. Further details and videos of modular paper sensor creation and 3D printing of the lid are in Supplementary Section 3, 4 .

2.2.2 Modular Pill Counter. The most important function of our system is to reliably count the number of pills coming out of the bottle and going into the bottle. We design the cap in such a way that pills can be accessed by tilting and shaking the container to allow the pills to come out of the slider, one at a time. First and foremost, our target application requires us to keep a log of all the pills coming out of the bottle and to detect an anomaly. In order to count the pills, we build a simple pill counter fitted inside the lid, using two sets of Infrared Light Emitting Diodes (IR LEDs) and a photodiode. When a pill passes between LED and detector, a change in voltage output confirms the presence of a pill passing through. Further details about the pill counter design and working principle can be found in Supplementary Section 5. The system also logs the number of pills being added into the container at the pharmacy, so that the system can keep an exact count of the number of pills in the container at all times.

\subsubsection{Modular Surveillance and Notification System.}

The 3D printed container lid with cap and pill health monitoring sticker placed inside the bottle can be seen in Figure 3a. As we are keeping track of the number of pills coming out and going into the container through the cap, we need to ensure there are no other available means to remove pills from the container, including by removing the cap or breaking the container. To detect an attempt to break the container, we use a Conductive Rubber Strain Sensor (CRSS). Our CRSS is a carbon-filled silicone which resistance (420 $\Omega / \mathrm{cm}$ ) depends on the length of the rubber cord. We use a $5 \mathrm{~cm}$ rubber cord embedded in a thin layer of Polydimethylsiloxane

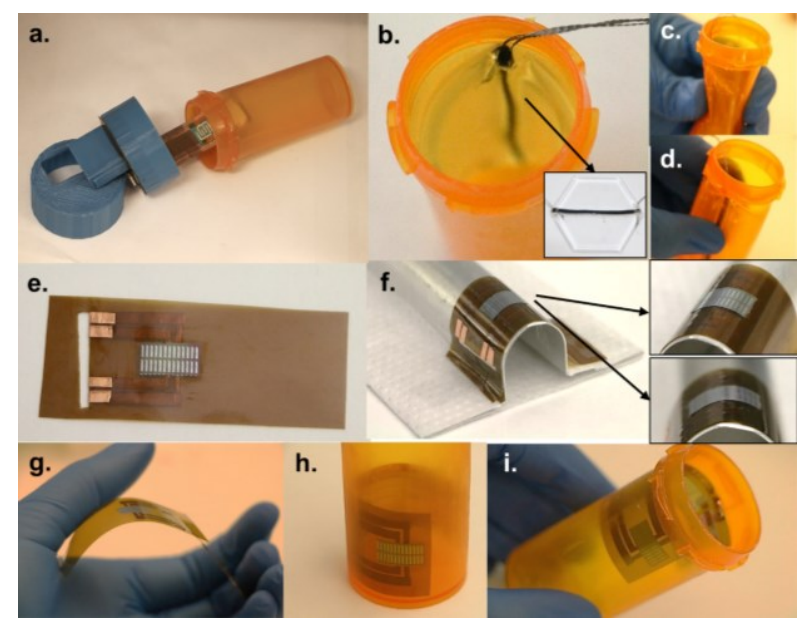

Figure 3: (a) Bottle with 3D printed lid and paper sensor sticker going inside the bottle. (b) A rubber strain sensor embedded in PDMS attached to the inside walls of the prescription container. (c) Holding the prescription container by hand. (d) Pressing the container as an imitation of tampering event changes the resistance of the strain sensor. (e) Flexible electronic interface decal having flexible silicon microprocessor with through-polymer-via connections to copper conductive tape on the backside. (f) Flexible electronic interface decal placed on a $1 \mathrm{~cm}$ bending radius platform with blow out image showing close-ups of the silicon microprocessor on the bent platform. (g) Flexible electronic interface decal attached to a paper sensor decal, bent and displayed in hands. (h) Flexible electronic interface decal with paper sensor decal inside a prescription container. (i) Slanted top view of a highlighting the capability of the decal interface to be placed anywhere inside the bottle.

(PDMS), and attached to the inner walls of the container (Figure 3b). PDMS is biocompatible and sticks to the inner walls of the container without the need for adhesives. The resistance of the rubber cord changes when it is stretched or bent. This change in resistance can be detected by our electronic interface and can generate an alert. To establish contact with the rubber cords, stainless steel conductive fibers are attached to both ends, with a knot. This provides a good contact to the stretchable rubber sensor, for it to be attached to the electronic interface. PDMS helps the sensor to be attached, inside the container, while allowing it to stretch at the same time. When someone tries to break the container (Figures $3 \mathrm{c}$ and $3 \mathrm{~d}$ ), the rubber cord bends and the electronic interface generates an alert, based on the change in resistance. Similarly, if someone removes the cap, the rubber sensor is stretched laterally as the contacts from the rubber sensor are moving up to the electronic interface located on the cap, and this stretching action produces a change in resistance that can again be identified by the electronic interface to generate an alert. In case the contact or the rubber stretch sensor breaks, the connection, in turn, gets broken; this is also sensed by the electronic interface, which generates an alert. The central electronic interface holds the key to recording all the activities and sending alerts. Without alerts, there is no way to record any discrepancy when someone tampers with the system. To prevent any mishandling of the electronic interface, we make a tamper sensor using paper-based sensors developed in our lab. It is a proximity-based pressure sensor to not only detect if someone touches the system but also to detect a hand that 
may come in close vicinity of the electronic interface. The tamper sensor was developed using the techniques presented by Nassar et al., using a parallel-plate capacitive sensor made of flexible aluminum foil for the metal plates, and a microfiber wipe placed in-between, to serve as a pressure sensitive dielectric. When pressure is applied to the sensor, the microfiber wipe compresses, changing the spacing between the two plates, which then results in a change in capacitance. The fabrication process is detailed in the publication by Nassar et al. [22] The sensor is converted into a modular decal sensor that can be integrated with the central electronic interface. A tamper sensor decal is attached directly above the electronic interface to log any approach or mishandling attempt.

\subsubsection{Modular Electronic Interface. Since we are using a}

DIY strategy to build the complete system, the central electronic interface has to be made according to the decal sensors we created in the previous sections. Our ACT based paper sensor integration approach makes it possible to fabricate in a modular fashion, any decal system that can be customized for a specific application. To build a fully conformal and flexible electronic interface for applications requiring complete flexibility, we turn to the flexible processes developed in our lab. Besides the flexible paperbased sensors introduced by Nassar et al., Sevilla et al have successfully demonstrated how bulk monocrystalline Silicon (100)-based high performance advanced Complementary Metal Oxide Semiconductor (CMOS) devices and circuitry can be flexed down to $0.5 \mathrm{~mm}$ bending radius $[23,24]$. One of our objectives is to embed the system inside the prescription container; to do so, we produce a fully flexible decal electronic interface. The flexible microprocessor acts as the digital logic component that takes analog data from the sensors, converts it to digital data and processes it to make decisions. The microprocessor feeds $200 \mathrm{~mA}$ current into the resistive temperature sensor and reads the voltage across the sensor which correlates to the temperature inside the bottle. For the humidity sensor, the processor feeds $3.3 \mathrm{~V}$ voltage across the capacitive structure. It notes down the time it takes to fully charge the capacitor which can be used to find the capacitance of the sensor and eventually find the relative humidity levels inside the prescription bottle. The flexible microprocessor provides an innate advantage of the ability to be interfaced with flexible sensors and be adhered to non-flat surfaces like that of the inside walls of a prescription bottle. The flexible microprocessor is attached on a polyimide sheet and connections to the copper interconnects are established on the back side, by through-polymer-vias (Figure 3e). The decal is placed on a $1 \mathrm{~cm}$ bending radius platform to demonstrate the overall flexibility of the full system (Figure 3f). Following the same integration approach described in Section 2.2.1, we attach the paper temperature and humidity sensors to this flexible electronic interface (Figure $3 \mathrm{~g}$ ). The complete system is then lined inside the prescription container so that it clasps to the inner walls of the container (Figure $3 \mathrm{~h}$ ). This DIY-based
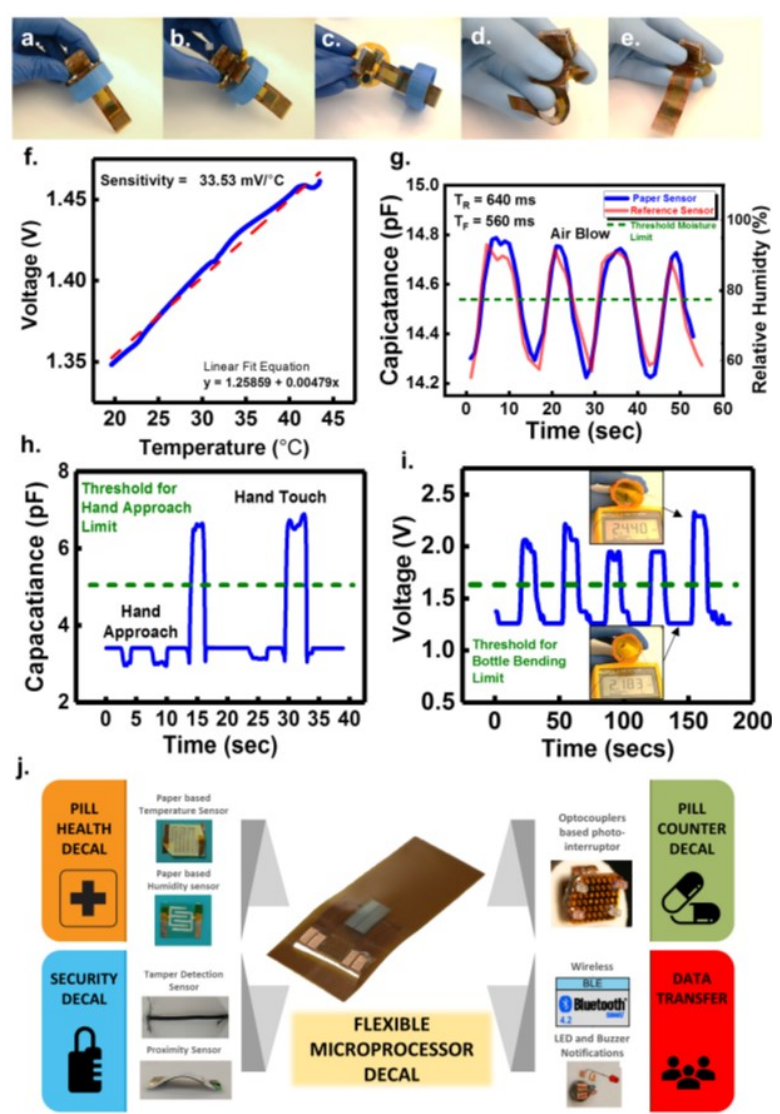

Time (sec)

Time (secs)

Figure 4: (a) Electronic interface decal held between fingers to remove it from the cap. (b) Electronic interface decal lifted with ease from its resting spot. (c) Electronic interface decal completely removed from the cap. (d) Electronic interface decal twisted between the fingers. (e) Electronic interface decal returned to its original form after twisting. (f) Temperature vs. Voltage for the paper temperature sensor. (g) Variations of capacitance in response to humidity changes testing using blowing (moist) human breath. (h) Capacitance output of the tamper sensor, with negative peaks for an approaching hand, and positive peaks for touching the electronic interface decal. (i) Voltage recording showing peaks when bottle is compressed. (j) A schematic overview of the decal based system.

system can monitor temperature and humidity inside the container (Figure 3i). Because it is light-weight and flexible, the system makes daily usage and handling of the container easy, while providing significant benefits to the patient. For wireless connectivity, we made an electronic system and its modular assembly detailed in Supplementary Section 6.

\section{Results and Discussion}

\subsection{Minimalistic Low-Cost Electronic Interface}

Our electronic interface encompasses major elements of an electronic system with, as main attributes have, a low power consumption, data transmission capability, and a small footprint. By using a flexible substrate (Polyimide) and sensors (Paper and rubber), and integrating them using anisotropic conductive tapes, we are able to design the complete assembly in any shape and functionality, as we want. For one specific application, we design a prototype to fit our $3 \mathrm{D}$ printed cap with a central electronic interface connected to 
paper temperature and humidity sensors and a buzzer. This customizability and flexibility allow us to easily install or remove the completely assembled unit from the cap, as shown in Figures $4 a-c$. As the substrate is very flexible, it can be easily folded and wrapped around to fit the shape of the container. Figures $4 \mathrm{~d}$ and $4 \mathrm{e}$ show the sensors being roughly handled by hands and the return to their original shape.

\subsection{Pill Health Monitoring and Surveillance Sensors}

Conventionally, changes in resistance are detected using a Wheatstone bridge, which uses 4 resistors connected to the battery, and separated, from the rest of the electronic interface. This consumes a significant amount of power, and thus a large battery is needed for long-term operation. The internal components of PSOC are used to make a current source, IDAC (Current Digital to Analog Converter), to feed a low amount of current directly to the resistive sensors (Supplementary Figure S3e). This source of current periodically distributes a fixed amount of current directly into the temperature and strain sensors, following which the subsequent voltage is read by an analog to digital converter (ADC) in the same PSOC. By limiting the amount of current, and feeding current only during the time periods when data are being logged in, we are able to reduce power consumption significantly. Conventionally, resistive sensors are used in a Wheatstone bridge powered by a battery at all times, for which the continuous current draw is $200-470 \mathrm{~mA}$. However, with our system, we only feed $600 \mu \mathrm{A}$ of current into the sensor and only when needed, i.e. once per second, which equates to energy savings of $99.7 \%$. Temperature-measuring resistive sensors have a small resistance change $\left(27 \mathrm{~m} \Omega /{ }^{\circ} \mathrm{C}\right)$ corresponding to only small voltage changes, consequently resulting in reduced sensitivity. We generally require the use of an amplifier IC, in the circuit, to enhance the sensitivity; this draws power of around $15-40 \mathrm{~mA}$, in addition to the power consumed by the Wheatstone bridge and the electronic interface. The advantage of the PSOC is further evidenced by our ability to enhance the sensitivity to $4.74 \mathrm{mV} /{ }^{\circ} \mathrm{C}$, using the PSOC's internal operational amplifier with a set gain of 1001 . A graph of the amplified voltage response from the sensor, using the PSOC, is shown in Figure 4f. The absolute temperature is measured by placing a commercial temperature sensor (Sensirion ${ }^{\circledR}$ SHTW2) close to the paper temperature sensor, and by heating up both sensors, using an incandescent lamp. The commercial sensor is used as a reference to compare the performance of our sensor. PSOC has a 10-bit $\mathrm{ADC}$ resolution and is able to sense a minimum voltage change of $1 \mathrm{mV}$. Thus, with our electronic interface, the temperature resolution is $0.22^{\circ} \mathrm{C}$. By taking advantage of the PSOC's features, we eradicate the need for an external signal conditioning interface and amplifiers to save power.

To monitor the humidity inside the prescription bottle, we use a paper-based humidity sensor. This sensor, as reported by Nassar et al. [22], changes the capacitance in response to changes in humidity. In order to monitor changes in capacitance, a capacitance-to-digital converter (CDC) IC is needed for the microcontroller to be able to measure the capacitance. This consumes extra power and space; however, we are able to use the inbuilt CapSense ${ }^{\circledR}$ feature in the PSOC to measure the capacitance without the need for any external components. The capacitance sensor is placed next to a commercial humidity sensor (Sensirion ${ }^{\circledR}$ SHTW2) and tested by blowing breath on both sensors. The results are reported in Figure $4 \mathrm{~g}$, which shows the changes in capacitance that are detected by the PSOC, in response to humidity, compared to the relative humidity measured by the commercial sensor. The commercial sensor was used to compare the performance of our sensor and relate the capacitance output of our sensor to relative humidity. A threshold is set, past which the system generates an alert that the humidity levels have become high. The responsivity of the sensor with the electronic interface comes out with $640 \mathrm{~ms}$ rise time $\left(\mathrm{T}_{\mathrm{R}}\right)$ and 540 fall time $\left(\mathrm{T}_{\mathrm{F}}\right)$, which is fast enough to report sudden humidity changes inside the container. Results in Figure 4h show that when the hand is brought close to the electronic interface with the tamper sensor attached, the capacitance decreases slightly due to proximity effects. To test this, we hover the hand $2 \mathrm{~cm}$ above the sensor. As soon as the hand touches the surface of the sensor, the sensor detects the pressure, resulting in a significant increase in capacitance, due to a decrease in the parallel plate spacing from the applied pressure [22]. The strain sensor attached to the walls of the bottle is a resistive sensor changing its resistance when subjected to strain. This concept is used to measure any forced entry attempt on the bottle. The same interface is shared between the strain sensor and the paper temperature sensor, where we inject a small amount of current in the resistive sensor and measure the resulting voltage. Figure $4 \mathrm{i}$ shows the voltage output from the strain sensor sensed by the electronic interface, with 2 snippets on the graph showing the change in resistance (measured using a digital multi-meter) observed when the bottle is pressed and released. Similar results are obtained when the cap is removed, as the pull on the strain sensor changes the resistance of the sensor. A threshold voltage level can be set, above which an alert can be generated by either using Bluetooth, logging in memory, lighting up the LED or sending an SMS using the GSM module. An overview of each sensor module's interface inside the PSOC and data transfer to the smartphone is shown in Supplementary Figure S2e.

\subsection{Pill Counter Demonstration}

The main objective of our device is to keep track of the number of pills taken and the alert user when it is time to take a pill (or several). The device was able to accurately count the number of pill coming in and going out of the container. Further details and a video demonstration can be found in Supplementary Section 7.

\section{Conclusion}


We have presented a Do-It-Yourself (DIY) modular integration strategy, using low-cost paper sensors and a flexible decal electronic interface that provide a complete turnkey solution for better adherence to prescribed medication. The device is designed to fit on a prescription container like any normal cap, with an additional height of only $2 \mathrm{~cm}$. The device keeps a $\log$ of pill intake and incorporates flexible paper-based sensors for various kinds of sensing. The data is synced continuously with a smartphone via Bluetooth. Additionally, we show a new DIY method of connecting the sensors with the electronic interface using an anisotropic conductive tape (ACT). The contact mechanics and electrical behavior are analyzed using FEM simulations to show how a pressure as little as finger pressure can successfully form a reliable electrical connection between two electrodes without the need for soldering or conductive epoxy. We show that the functioning of the device, while connected to the drug container, displays reliable and consistent results. The integration process involves the assembly of low-cost materials without the need for complex fabrication processes. It has the ability to count pills, going in and out of the container; it can also detect forced entry attempts on the container and electronic interface tampering attempts. It monitors the temperature and humidity inside the container, sends alerts using Bluetooth, and turns on a buzzer/LED. Figure $4 \mathrm{j}$ shows a schematic of the various components of the smart lid. This device has tremendous potential to save human lives and billions of dollars for the US healthcare system.

\section{Acknowledgments}

This publication is based upon work supported by the King Abdullah University of Science and Technology (KAUST) Office of Sponsored Research (OSR) under Award No. REP/1/2880-01-01 KFUPM.

\section{References}

1. Rep, M.M.W., Battling the biology of opioid addiction. Morb. Mortal. Wkly. Rep, 2016. 65: p. 1445-1452.

2. Nam, Y.H., et al., State prescription drug monitoring programs and fatal drug overdoses. The American journal of managed care, 2017. 23(5): p. 297.

3. Hall, A.J., et al., Patterns of abuse among unintentional pharmaceutical overdose fatalities. Jama, 2008. 300(22): p. 2613-2620.

4. Peirce, G.L., et al., Doctor and pharmacy shopping for controlled substances. Medical care, 2012. 50(6): p. 494500 .

5. Cattell, V., Poor people, poor places, and poor health: the mediating role of social networks and social capital. Social science \& medicine, 2001. 52(10): p. 1501-1516.

6. Lynch, J.W., G.A. Kaplan, and J.T. Salonen, Why do poor people behave poorly? Variation in adult health behaviours and psychosocial characteristics by stages of the socioeconomic lifecourse. Social science \& medicine, 1997. 44(6): p. 809-819.

7. Chang, S.-M., et al., Characteristic study of anisotropicconductive film for chip-on-film packaging. Microelectronics Reliability, 2001. 41(12).
8. Watanabe, I., et al. Recent advances of interconnection technologies using anisotropic conductive films in flat panel display applications. in Advanced Packaging Materials: Processes, Properties and Interfaces, 2004. Proceedings. 9th International Symposium on. 2004. IEEE.

9. Yim, M.J. and K.W. Paik, Recent advances on anisotropic conductive adhesives (ACAs) for flat panel displays and semiconductor packaging applications. International journal of adhesion and adhesives, 2006. 26(5): p. 304-313.

10. Astin, A.D., Finger force capability: measurement and prediction using anthropometric and myoelectric measures. 1999, Virginia Tech.

11. Sletbak, J., et al., Glowing contact areas in loose copper wire connections. IEEE transactions on components, hybrids, and manufacturing technology, 1992. 15(3): p. 322-327.

12. Nassar, J.M., et al., Compliant lightweight non-invasive standalone "Marine Skin" tagging system. npj Flexible Electronics, 2018. 2(1): p. 13.

13. Nassar, J.M., et al., Compliant plant wearables for localized microclimate and plant growth monitoring. npj Flexible Electronics, 2018. 2(1): p. 24.

14. Khan, S.M., et al., Flexible Lightweight CMOS-Enabled Multisensory Platform for Plant Microclimate Monitoring. IEEE Transactions on Electron Devices, 2018. 65(11): p. 5038-5044.

15. Pavlovska, G. and S. Tanevska, Influence of temperature and humidity on the degradation process of ascorbic acid in vitamin $C$ chewable tablets. Journal of thermal analysis and calorimetry, 2013. 111(3): p. 1971-1977.

16. Rangaiah, K., S.C. Chattaraj, and S.K. Das, Effects of solvents, temperature, and plasticizer on film coating of tablets. Drug development and industrial pharmacy, 1997. 23(4): p. 419-423.

17. Viljoen, J.M., et al., Effect of moisture content, temperature and exposure time on the physical stability of chitosan powder and tablets. Drug development and industrial pharmacy, 2014. 40(6): p. 730-742.

18. Takekuma, Y., et al., Difference in the Dissolution Behaviors of Tablets Containing Polyvinylpolypyrrolidone (PVPP) Depending on Pharmaceutical Formulation After Storage Under High Temperature and Humid Conditions. Journal of Pharmacy \& Pharmaceutical Sciences, 2016. 19(4): p. 511-519.

19. Nakamura, Y., et al., Influence of temperature and humidity on physico-pharmaceutical characteristics of Rasilez (®) tablets. Yakugaku zasshi: Journal of the Pharmaceutical Society of Japan, 2014. 134(4): p. 555-561.

20. Wang, J.T., et al., Effects of humidity and temperature on in vitro dissolution of carbamazepine tablets. Journal of pharmaceutical sciences, 1993. 82(10): p. 1002-1005.

21. David, S. and C. Gallian, The effect of environmental moisture and temperature on the physical stability of effervescent tablets in foil laminate packages containing minute imperfections. Drug Development and Industrial Pharmacy, 1986. 12(14): p. 2541-2550.

22. Nassar, J.M., et al., Paper skin multisensory platform for simultaneous environmental monitoring. Advanced Materials Technologies, 2016. 1(1).

23. Rojas, J.P., et al., Transformational silicon electronics. ACS nano, 2014. 8(2): p. 1468-1474.

24. Torres Sevilla, G.A., et al., Flexible nanoscale highperformance FinFETs. ACS nano, 2014. 8(10): p. 98509856. 